\title{
The Effects of Ascorbic Acid and Garlic on Bone Mineralization in Lead Exposed Pregnant Rats
}

\author{
Alireza Ebrahimzadeh-Bideskan ${ }^{1}$; Akram Sadeghi ${ }^{1}$; Fatemeh Alipour ${ }^{1}$; Mojtaba Kianmehr ${ }^{2, *}$ \\ ${ }_{1}^{1}$ Department of Anatomy and Cell Biology, Faculty of Medicine, Mashhad University of Medical Sciences, Mashhad, Iran \\ ${ }^{2}$ Department of Medical Physics, Faculty of Medicine, Gonabad University of Medical Sciences, Gonabad, Iran \\ ${ }^{*}$ Corresponding author: Mojtaba Kianmehr, Corresponding author at: Gonabad University of Medical Sciences, Gonabad, Iran., E-mail: kianmehr.m@gmu.ac.ir
}

Received: January 31, 2014; Accepted: May 2, 2014

\begin{abstract}
Background: Lead exposure during pregnancy may impair skeletal development. Oxidative stress is one of the important mechanisms for lead toxicity effects. The aim of this study was to investigate ascorbic acid and garlic effects on bone mineralization in lead exposed pregnant rats.

Materials and Methods: In this experimental study, 50 pregnant Wistar rats were randomly divided into 5 groups; group (L) exposed to lead acetate, group $(\mathrm{L}+\mathrm{C})$ exposed to lead acetate and ascorbic acid (vitamin $\mathrm{C}$ ), group $(\mathrm{L}+\mathrm{G})$ exposed to lead acetate and garlic juice, sham group treated with tap water plus $0.4 \mathrm{~mL} / \mathrm{L}$ normal hydrogen chloride $(\mathrm{HCl})$ and $0.5 \mathrm{mg} / \mathrm{L}$ sugar, control group without any intervention. All treatments were done during pregnancy. After birth, blood and bone lead levels were measured and then all neonates were sacrificed, and their right tibia bone processed for alizarin red and Alcian blue staining.

Results: Blood lead levels in L group increased significantly in both mothers and their neonate compared to control animals. In addition, the neonates born to L group showed markedly higher lead concentrations in their bone than that of controls. In contrast, we found no significant changes in blood and bone lead levels in lead exposed neonates that received ascorbic acid and garlic. Bone formation in neonates of L group was clearly disrupted. Interestingly, both ascorbic acid and garlic treatments could apparently improve bone formation during pregnancy in lead exposed neonates.

Conclusion: Ascorbic acid and garlic consumption during pregnancy may improve the deleterious effects of lead exposure on bone mineralization.
\end{abstract}

Keywords:Lead acetate; Bone mineralization; Ascorbic acid; Garlic; Rat neonates

\section{Introduction}

Lead is a ubiquitous environmental pollutant that even in small quantities produces wide range of toxic effects in man and in experimental animals [1-3]. It is well known that lead accumulates in the skeleton throughout development and localizes in areas of bone mineralization and growth [4]. There are strong clinical evidence linking to lead exposure during intrauterine life may result in reduced birth weight, decreased preadolescent growth rates, and impaired skeletal development $[4,5]$.

Several in vivo and in vitro investigations showed that lead inhibits bone formation, delays growth plate chondrocyte maturation, and inhibits mineralization during ectopic bone induction [4-6]. Kennedy et al. demonstrated that lead could cause bone malformations in the rat and mouse fetuses [7].

However, the exact mechanisms underlying these effects remain unclear; many hypotheses have been given to explain lead toxicity in skeletal growth such as: impairment in the hormonal regulation of calcium absorption, displacement with calcium in the mineral bone matrix, direct effects on osteoblast function, inhibition of the vi- tamin D3 stimulated synthesis of osteocalcin and oxidative stress produce induction [8].

The proposed mechanism for lead induced oxidative stress is addressing by its role in the generation of ROS (Reactive Oxygen Species), plus its effect on the antioxidant defense system $[9,10]$. Therefore, reducing the possibility of lead interacting with critical biomolecules and bolstering the cell's antioxidant defenses might be associated with the beneficial role of antioxidant nutrients through exogenous supplementation of antioxidant molecules [11, 12].

Ascorbic acid (vitamin C) is a low molecular mass antioxidant that scavenges the aqueous ROS by very rapid electron transfer that inhibits lipid peroxidation [13]. In the previous works ascorbic acid supplementation in lead exposed animals significantly reduced blood, liver and renal lead levels [9-12]. Early reports found that ascorbic acid might act as a possible chelator of lead, with similar potency to that of (ethylene diamine tetraacetic acid) EDTA. Although, there has been considerable debate concerning the relationship between ascorbic acid 
nutritional status and heavy metal body burden in lead induced toxic effects $[10,11]$.

Today, interest in using of medicinal plants because of their wide range of efficacy and lesser side effects has been increased as a health aid. Garlic (Allium sativum L.) as a medicinal plant has been reported to contain two main classes of antioxidant components, namely flavonoids, and sulfur containing compounds (diallyl sulfide, trisulfide and allyl-cysteine). These are likely to play an important role in the widely demonstrated therapeutic and antioxidant effects. Besides these properties, the other efficiency of garlic is perhaps due to the presence of these sulfur-containing amino acids and compounds having free carboxyl $(\mathrm{C}=\mathrm{O})$ and amino $\left(\mathrm{NH}_{2}\right)$ groups in their structures [14-16]. These biologically active compounds might chelate lead and enhance its excretion from the body resulting in reduced lead accumulation in soft tissues and blood [17].

Therefore, this work was designed in order to clarify the beneficial effects of ascorbic acid and garlic as two antioxidants during pregnancy on the bone mineralization of prenatally lead exposed rat neonates.

\section{Materials and Methods}

This experimental study was done in Mashhad University of Medical Sciences according to ethics committee guide lines and all protocols of animal experiments have been approved by the Institution's Animal Care Committee.

Animals: Fifty virgin adult female Wistar rats (8-10 weeks age, weighting between 250-300 g) were obtained from the Faculty of Medicine Animal Lab, Mashhad University of Medical Sciences and used in this study. The animals were housed in an environmentally controlled room (12 $\mathrm{h} / 12 \mathrm{~h}$ light and dark cycle, $22^{\circ} \mathrm{C}$ temperature and $60 \%$ relative humidity) in individual cages. All animals were received laboratory chow and water ad libitum.

The female rats were mated overnight with fertile males of the same strain ( 1 male rat and 3 female ones in each cage). Vaginal smears were taken from mated females in the next morning and pregnancy was determined by the presence of spermatozoa in the smears, which denote the embryonic day zero $\left(\mathrm{E}_{0}\right)$. The pregnant female rats were randomly divided into 5 groups as follows.

Group I(L): Includes pregnant rats, were exposed to lead acetate in the drinking water (1500 ppm) during pregnancy from $\mathrm{E}_{0}$ to $\mathrm{E}_{21}$. Group II $(\mathrm{L}+\mathrm{C})$ : Includes pregnant rats, which were exposed to lead acetate in the drinking water $(1500 \mathrm{ppm})+500 \mathrm{mg} / \mathrm{kg}$ vitamin C (i.p.) once a day, during pregnancy from E0 to E21.Group III $(L+G)$ : Includes pregnant rats, which were exposed to lead acetate in the drinking water (1500 ppm) $+1 \mathrm{~mL}$ garlic juice (daily dose of $400 \mathrm{mg} / \mathrm{kg}$ by gavage), during pregnancy from $\mathrm{E}_{0}$ to $\mathrm{E}_{21}$. Group IV (Sham): Includes pregnant rats, which administrated with tap water+0.4 mL/L $\mathrm{HCl}$ and $0.5 \mathrm{mg} / \mathrm{L}$ glucose, during pregnancy from $\mathrm{E}_{0}$ to $\mathrm{E}_{21}$. Group $\mathrm{V}$ (control): Includes pregnant rats, which administrated with drinking water without any intervention.Garlic Juice Preparation: Fresh garlic (Allium sativum L.) bulbs were purchased from the local market in Mashhad, Iran. Then, they were identified at The Department of Botany, Ferdowsi University of Mashhad herbarium of Mashhad (FUMH, NO: 39493), Iran. Garlic bulbs were peeled and washed with distilled water. After drying in a shed, about $500 \mathrm{~g}$ of clean garlic bulb were crushed with the help of an electric grinder.

The resultant was squeezed and filtered through a fine cloth and the filtrate was quickly frozen until used. The concentration of the juice was calculated as [18]:

Gram = Weight of fresh garlic/Weight of the juice

Leaded water preparation: Lead acetate was purchased from Merck (Germany). For preparation of 1500 ppm leaded water: $30 \mathrm{~g}$ lead acetate $+8 \mathrm{~mL} \mathrm{HCl}+10 \mathrm{~g}$ glucose solved in $20 \mathrm{~L}$ of drinking water.

To prevent the formation of lead precipitation, normal $\mathrm{HCl}$ and to improve taste of leaded drinking water, glucose was added to solution $[19,20]$.

Whole blood and bone lead concentrations measurement: Blood samples from the female rats and their offspring were drawn into glass tubes that had been treated with heparin. The tubes were inverted several times for mixing heparin and blood to prevent blood coagulation. In addition, the lead contents of the neonate's left tibia were examined.

To measure lead level in whole blood and bone samples, a Perkin-Elmer Model 3030 atomic absorption spectrophotometer with a Perkin-Elmer HGA (heat graphite atomizer) 400 graphite furnace and hydride system MHS 10 were used together with HCL (hallow cathode lamp) and EDL (electrode discharge lamp) for metal measurement even in very low levels.

At the end of pregnancy, the animals were allowed to deliver naturally; and then their neonates at first postnatal day were anesthetized with chloroform and divided in two categories randomly for using tow staining methods as follow:

1-Whole body preparation: Four whole bodies of neonates in each group were fixed in alcohol (95\%) for a week and then immersed in acetone. The spacemen were stained with alizarin red and Alcian blue for 5 days at $37^{\circ} \mathrm{C}$. After staining, the spacemen were washed in distilled water. In order to remove soft tissues, the samples were placed in glycerine $20 \%$ containing $\mathrm{KOH} 1 \%$ for a week and then immersed in glycerine 50\% plus $\mathrm{KOH} 1 \%$ for a week. Finally the spacemen were put and saved in pure glycerine. For alizarin red and Alcian blue dye preparation $0.3 \%$ Alcian blue was dissolved in alcohol $70^{\circ}$ (1 volume), alizarin red $0.1 \%$ was dissolved in alcohol $95^{\circ}$ ( 1 volume), acid acetic ( 1 volume) and alcohol $70^{\circ}$ (17 volume). At the end of staining process, the mineralization zones of neonate's skeleton were become red and cartilage tissues become blue. Finally, the stained samples were photographed by means of apple 5 phone camera.

2-Tissue Preparation: Four pups per each group were used and their right tibia bones dissected carefully and stored in $10 \%$ buffered formalin for several days. After fixa- 
tion, the samples were dehydrated in sequentially increasing concentrations of ethanol followed by xylene, and embedded into paraffin. Then, tissue blocks were cut into 5 $\mu \mathrm{m}$ thickness and processed for histochemical studies using alizarin red and Alcian blue staining. The sections were scanned and photographed using a light microscope.

Statistical Analysis: Data were analyzed by the SPSS-15 statistical software (SPSS Inc., Chicago, IL). The differences between pre and post treatment in each group were determined using paired samples t-test and differences between all groups were assessed by one-way analysis of variances (ANOVA) followed by Tukey test. Results were expressed as means and were regarded as being significant at $\mathrm{p}<0.05$.

\section{Results}

There was no significant difference in maternal blood lead levels in different groups before starting the experiments. The bone and blood lead levels were significantly higher in the lead exposed (Group L) mother and their offspring ( $\mathrm{p}<0.001$, Fig. 1). However, in comparison to controls, we found no significant differences in blood lead levels in mothers and their neonates that received leaded water with vitamin $\mathrm{C}(\mathrm{L}+\mathrm{C})$ and garlic juice $(\mathrm{L}+\mathrm{G})$ (Fig. 2). The bone lead concentrations in lead exposed group born neonates also were significantly higher than that of controls ( $\mathrm{p}<0.001$, Fig. 3 ).

Whole embryos study: Our results demonstrated that the whole neonate staining intensity in lead exposure group was weak (pale red) comparing to other groups that was intensive (red) (Fig. 4).

Histological Study: To assess the effects of prenatally lead exposure on bone mineralization deficits in neonates, and the beneficial effects of ascorbic acid and garlic juice consumption on this process, we used alizarin red - Alcian blue staining. Because of the bones of lead exposed group offspring showed a lower mineralization, and the cartilage tissue background was observed. Therefore, in microscopic study of bone sections, neonates' bones of the lead exposed group demonstrated uncalcified cartilage content more than controls showing an abnormal osteogenesis and retarded bone formation in offspring born to lead exposed mothers. In contrast, the bone formation in the neonates of lead exposed group that received vitamin $C(L+C)$ and garlic juice $(L+G)$ showed normal ossification (Fig. 5).

\section{Discussion}

Results of this study showed that the bone and blood lead levels were significantly higher in the lead exposed mother and their offspring. There was no significant difference in blood and bone lead levels in mothers and their neonates that received leaded water with vitamin $C$ and garlic juice. In microscopic study of neonates bones in lead exposed group demonstrated uncalcified cartilage content more than control group. In contrast, the bone formation in the neonates of lead exposed group that received vitamin $\mathrm{C}$ and garlic juice showed normal ossification.

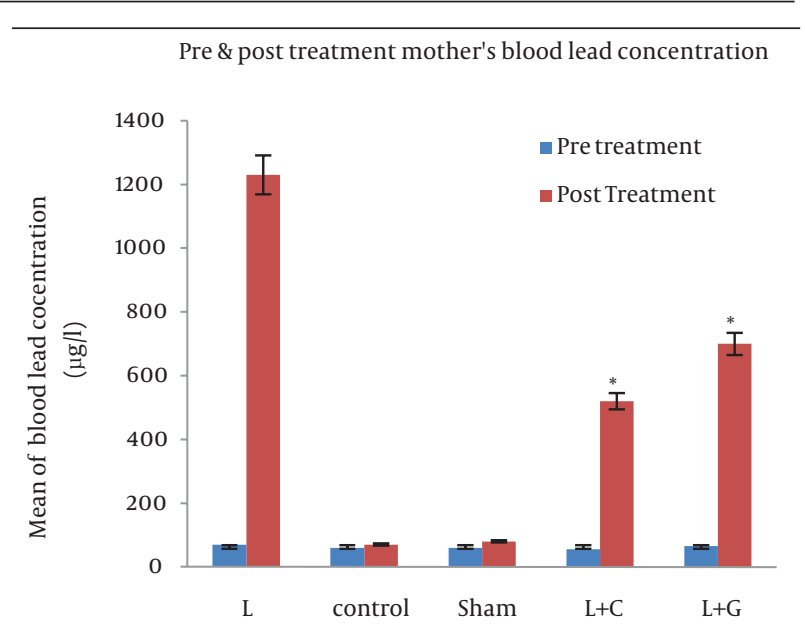

$\overline{\text { Figure 1. Maternal blood lead concentrations of different studied groups, }}$ maternal blood lead levels are showed at the pretreatment and the post treatment; data indicate statistically significant differences between lead exposed group's mothers with control, sham, lead+vitamin C ( + +C), and lead+garlic $(L+G)$ groups $(\mathrm{p}<0.001)$. Data are expressed as mean. ${ }^{*} \mathrm{p}<0.05$ compared with lead-treated group

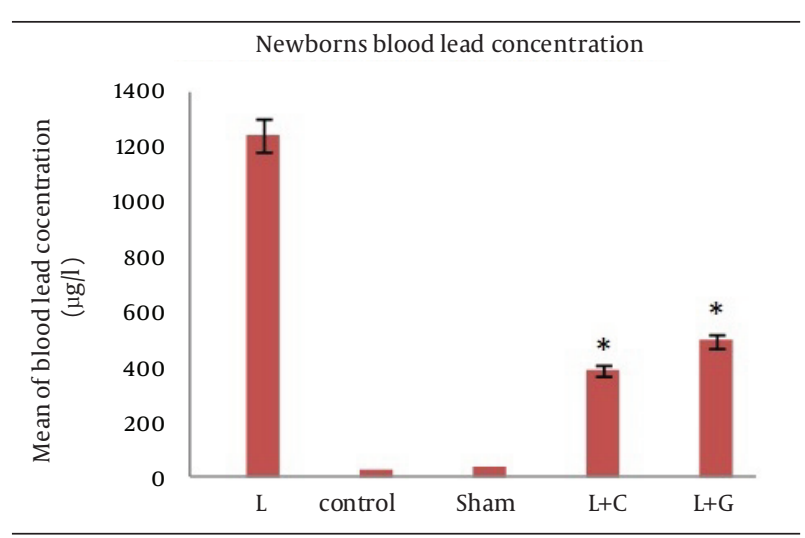

Figure 2. Blood lead concentrations of neonates in different studied groups, data indicate statistically significant differences between lead exposed group's neonates with control, sham, lead+vitamin $\mathrm{C}(\mathrm{L}+\mathrm{C})$, and lead+garlic $(\mathrm{L}+\mathrm{G})$ groups $(\mathrm{p}<0.001)$. Data are expressed as mean. ${ }^{*} \mathrm{p}<0.05$ compared with lead-treated group

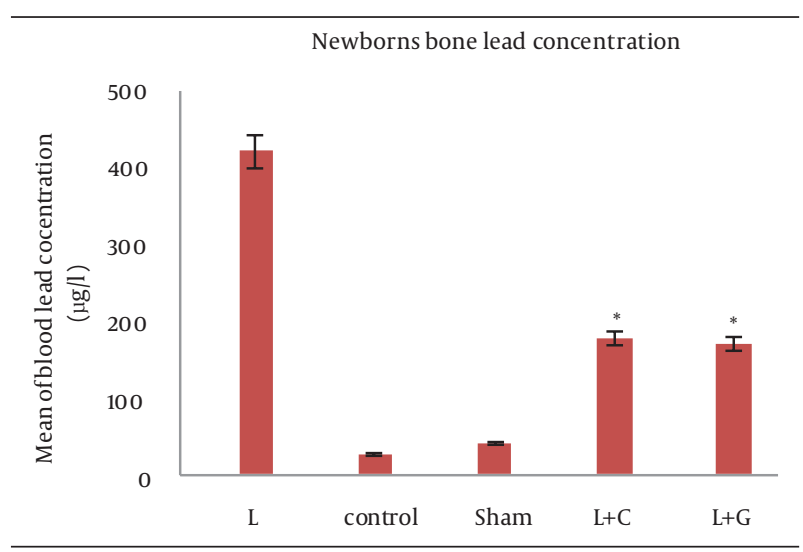

Figure 3. Bone lead concentrations of neonates in different studied groups, data indicate statistically significant differences between lead exposed group's neonates with control, sham, lead+vitamin C ( + +C), and lead+garlic $(\mathrm{L}+\mathrm{G})$ groups $(\mathrm{p}<0.001)$. Data are expressed as mean. ${ }^{*} \mathrm{p}<0.05$ compared with lead-treated group 
Ebrahimzadeh-Bideskan A et al.
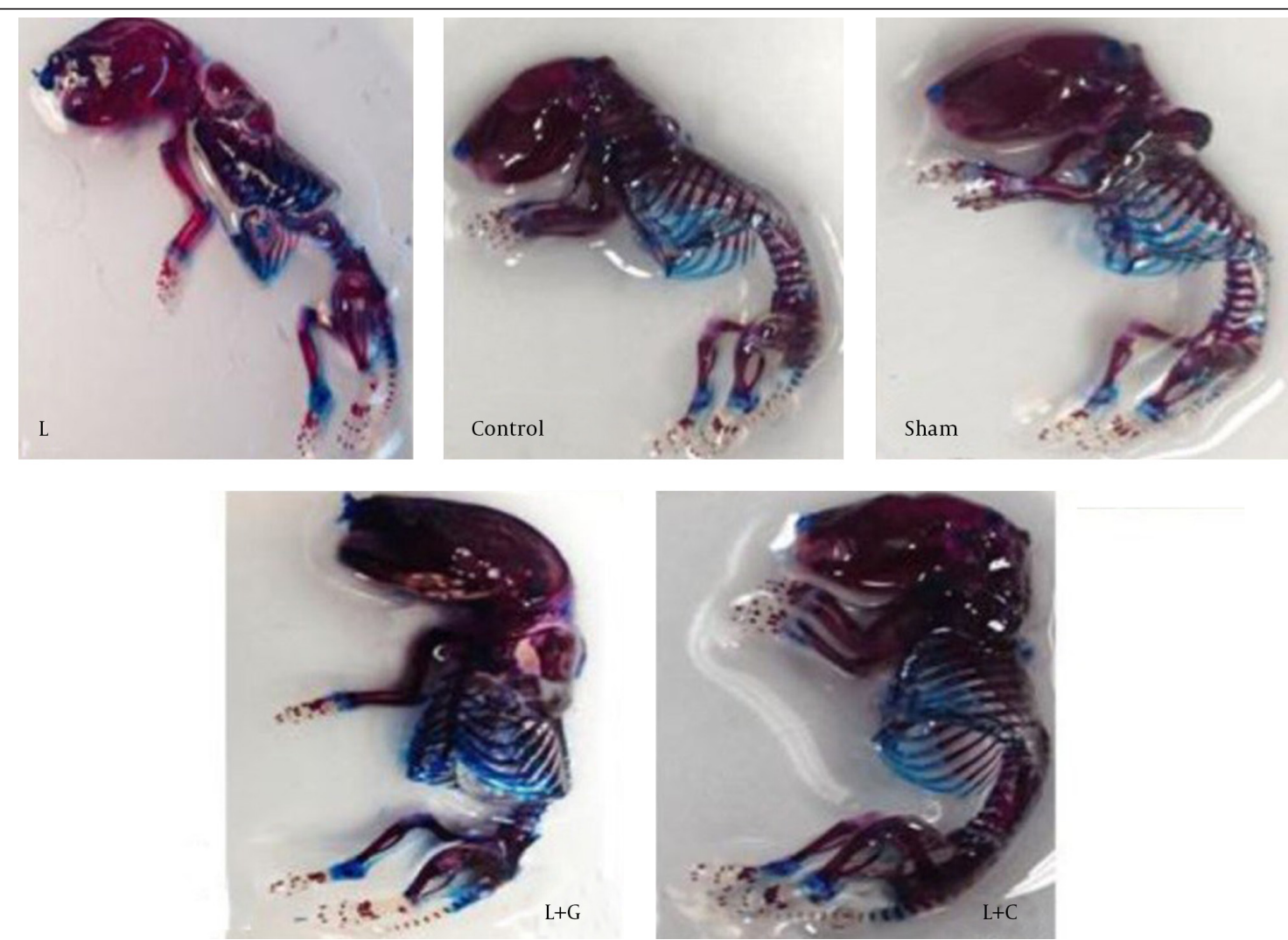

Figure 4. Photographs of whole neonates in different studied groups that stained with alizarin red - Alcian blue; lead exposed (L), control, sham, lead+garlic $(\mathrm{L}+\mathrm{G})$ and lead+vitamin $\mathrm{C}(\mathrm{L}+\mathrm{C})$ groups
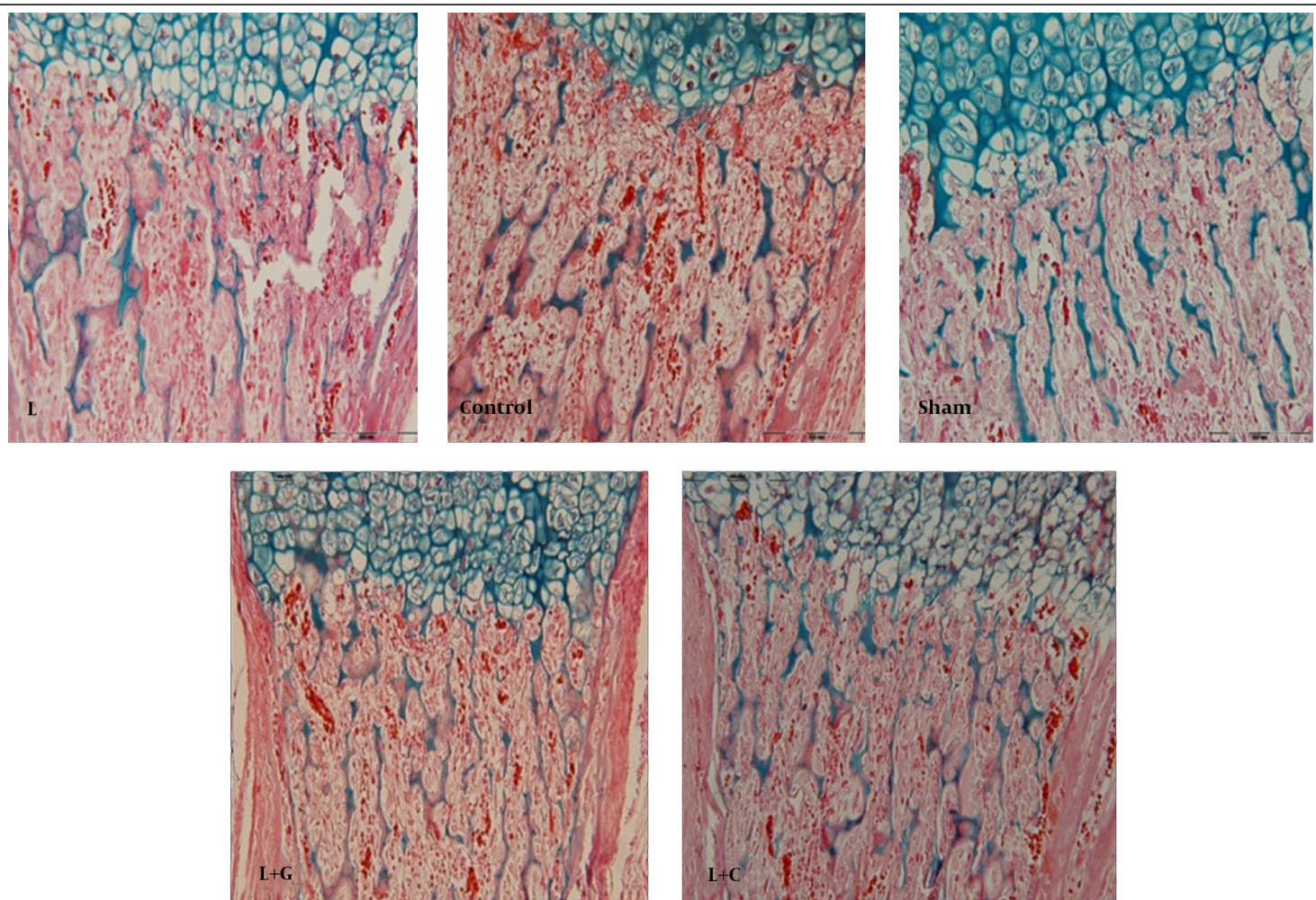

Figure 5. Photomicrographs of neonates tibia's bones in different studied groups that stained with Alizarin red-Alcian blue. EC; Epiphyseal cartilage, B; forming bone, Magnification 40×, lead exposed (L), control, sham, lead+garlic (L+G) and lead+vitamin C (L+C) groups 
Previous human and animal investigations indicated that the prenatally lead exposure suppresses growth processes. An important element of somatic growth is skeletal growth.

The fetal skeleton is a useful indicator of embryonic development and frequently reflects changes in the maternal-fetal environment [21, 22]. Aliverti et al. studied the number of ossification centres in different skeletal districts of rat fetuses, and suggested that the stage of skeletal ossification can be used in teratogenic studies in the rat to evaluate retarded fetal development [22]. The degree of growth retardation also was found to differ among ossification centers [23].

The results of present study indicated the leaded water consumption could successfully elevate the whole blood lead concentrations in both mothers and their neonates. Because lead crosses through the placental barrier freely, it will be logical if maternal lead exposure during pregnancy increases lead levels in neonates. Our results confirm the previously human and animal studies reported findings that maternal lead burden is an important determinant of infant lead levels. It is necessary to note that this observation was relevant to human pregnancy because most women are likely to have limited exposure to lead during pregnancy, but may have considerable body lead burdens from a history of lead exposure, including exposure during childhood [23-26].

Our data also showed significantly higher lead contents in bone of neonates born to lead exposed rats than that of controls. It is well documented that lead has the affinity to accumulate in the bone throughout development, and has direct and indirect effects on growth. The mechanisms behind lead effects on growth seem to involve actions at different sites. Lead may affect directly on osteoblast function, interfere with vitamin D metabolism or with calcium's role as a cellular messenger in its endocrine functions, or it may act as a depressant of food intake $[6,8,23,24]$.

The histological results in our study showed an apparent defect in ossification of growth cartilage plates in neonates that exposed with lead in prenatal period. These observations add to the recent accumulating evidence suggesting that intrauterine lead exposure inhibits bone formations in experimental animals. Many previous studies have shown that the lead localizes in areas of bone mineralization and can cause bone malformations in the rat and mouse fetuses, delay growth plate chondrocyte maturation, inhibit bone formation in dogs, and inhibit mineralization in vivo during ectopic bone induction $[6,7,24]$.

The present study results indicated that the lead concentrations of whole blood and bone in neonates born to lead exposed rats that received ascorbic acid and garlic juice showed insignificantly difference when compared to controls. Moreover, we can not found any histological changes in bone formation in neonates born to lead exposed rats that received ascorbic acid and garlic juice.
In agreement with the other studies, these results demonstrated that antioxidants (i.e. ascorbic acid and garlic) may prevent the lead cross from placental barrier, and this role may be reflect in their preventive effects on bone malformation.

Recent studies have suggested oxidative stress as one of the important mechanisms of toxic effects of lead. So, antioxidant defenses have been found to be defective in many of the disturbances caused by ROS production [10, 25].Ascorbic acid is considered one of the most prevalent antioxidative components of fruits and vegetables that interact directly with the oxidizing radicals. Ascorbic acid also is essential in collagen formation and ascorbic acid deficiency results in an abnormal extracellular matrix of the connective tissues, and a disrupted endochondral ossification [26].

Garlic is also a good source of phytochemicals with proven antioxidant properties. The biological activity of garlic may be mediated by its organic allyl sulfur components (mainly Allicin). It has been suggested that the biological activities of these ingredients may be related to their thiol modification and antioxidant characteristics [26].

In conclusion, the present study demonstrated that consumption of both ascorbic acid and garlic juice throughout pregnancy has beneficial roles on the lead induced toxicity on rat neonate's bones. These supplements had preventive effects not only in elevated blood and bone lead concentrations but also in bone formation deficiencies. Although, this idea need to more investigations, the authors recommend the consumption of plenty of raw garlic within the food as well as utilization of fresh fruits rich of ascorbic acid, especially for pregnant women living in lead contaminated areas such as industrial and central urban points to protect themselves and their offspring against any expected harms such as skeletal defects.

\section{Acknowledgements}

The authors express their gratitude for the approval of this research proposal (registration No. 89230) to the Research Deputy of the Mashhad University of Medical Sciences, Mashhad, Iran. The authors also wish to thank the Research Deputy of the Gonabad University of Medical Sciences, Gonabad, Iran for the support of the research proposal (registration No.1-369).

\section{Authors' Contributions}

All authors had equal role in design, work, statistical analysis and manuscript writing.

\section{Conflict of Interest}

The authors declare no conflict of interest.

\section{Funding/Support}

Mashhad and Gonabad Universities of Medical Sciences 


\section{References}

1. Sadeghi A, Ebrahimzadeh Bideskan A, Alipour F, Fazel A, Haghir $\mathrm{H}$. The Effect of Ascorbic Acid and Garlic Administration on LeadInduced Neural Damage in Rat Offspring's Hippocampus. Iran J Basic Med Sci. 2013;16(2):157-64.

2. Lodi S, Sharma V, Kansal L. The protective effect of Rubia cordifolia against lead nitrate-induced immune response impairment and kidney oxidative damage. Indian J Pharmacol. 2011;43(4):441-4.

3. Poreba R, Gac P, Poreba M, Andrzejak R. Environmental and occupational exposure to lead as a potential risk factor for cardiovascular disease. Environ Toxicol Pharmacol. 2011;31(2):267-77.

4. Ronis MJ, Aronson J, Gao GG, Hogue W, Skinner RA, Badger TM, et al. Skeletal effects of developmental lead exposure in rats. Toxicol Sci. 2001;62(2):321-9.

5. Saleh HA, El-Aziz GA, El-Fark MM, El-Gohary M. Effect of maternal lead exposure on craniofacial ossification in rat fetuses and the role of antioxidant therapy. Anat Histol Embryol. 2009;38(5):392-9.

6. Hamilton JD, O'Flaherty EJ. Influence of lead on mineralization during bone growth. Fundam Appl Toxicol. 1995;26(2):265-71.

7. Kennedy GL, Arnold DW, Calandra JC. Teratogenic evaluation of lead compounds in mice and rats. Food Cosmet Toxicol. 1975;13(6):629-32.

8. Pounds JG, Long GJ, Rosen JF. Cellular and molecular toxicity of lead in bone. Environ Health Perspect. 1991;91:17-32.

9. Adonaylo VN, Oteiza PI. Lead intoxication: antioxidant defenses and oxidative damage in rat brain. Toxicol. 1999;135(2-3):77-85.

10. Hsu P. Antioxidant nutrients and lead toxicity. Toxicol. 2002;180(1):33-44.

11. Vij AG, Satija NK, Flora SJ. Lead induced disorders in hematopoietic and drug metabolizing enzyme system and their protection by ascorbic acid supplementation. Biomed Environ Sci. 1998;11(1):7-14.

12. Jones DP, Kagan VE, Aust SD, Reed DJ, Omaye ST. Impact of nutri ents on cellular lipid peroxidation and antioxidant defense system. Fundam Appl Toxicol.1995;26(1):1-7.

13. Akbari A, Jelodar G, Nazifi S. The prophylactic effect of vitamin $\mathrm{C}$ on oxidative stress indexes following exposure to radio frequency wave generated by a BTS antenna model in rat liver and kidney. Zahedan J Res Med Sci. 2014;16(2):19-23.

14. Sharma V, Sharma A, Kansal L. The effect of oral administration of Allium sativum extracts on lead nitrate induced toxicity in male mice. Food Chem Toxicol. 2010;48(3):928-36.
15. Kodera Y, Suzuki A, Imada O, Kasuga S, Sumioka I, Kanezawa A et al. Physical, chemical, and biological properties of s-allylcysteine, an amino acid derived from garlic. J Agric Food Chem. 2002;50(3):622-32.

16. Taji F, Shirzad H, Ashrafi K, Parvin N, Kheiri S, Namjoo A, et al. A comparison between the antioxidant strength of the fresh and stale Allium sativum (garlic) extracts. Zahedan J Res Med Sci. 2012;14(5):25-9.

17. Crowe A, Morgan EH. Interactions between tissue uptake of lead and iron in normal and iron-deficient rats during development. Biol Trace Elem Res. 1996;52(3):249-61.

18. Senapati SK, Dey S, Dwivedi SK, Swarup D. Effect of garlic (Allium sativum L.) extract on tissue lead level in rats. J Ethnopharmacol. 2001;76(3):229-32.

19. Han JM, Chang BJ, Li TZ, Choe NH, Quan FS, Jang BJ, et al. Protective effects of ascorbic acid against lead-induced apoptotic neurodegeneration in the developing rat hippocampus in vivo. Brain Res. 2007;1185:68-74.

20. Nematbakhsh M, Rajabi P, Samarian SH. [The effect of lead on endothelial permeability of aorta] Persian. Razi J Med Sci. 2001;8(23):76-83.

21. Ronis MJ, Gandy J, Badger T. Endocrine mechanisms underlying reproductive toxicity in the developing rat chronically exposed to dietary lead. J Toxicol Environ Health A. 1998;54(2):77-99.

22. Aliverti V, Bonanomi L, Giavini E, Leone VG, Mariani L. The exten of fetal ossification as an index of delayed development in teratogenic studies on the rat. Teratology. 1979;20(2):237-42.

23. Hammond PB, Chernausek SD, Succop PA, Shukla R, Bornschein RL. Mechanisms by which lead depresses linear and ponderal growth in weanling rats. Toxicology and Applied Pharmacology. 1989;99(3):474-86.

24. Armocida A, Beskow P, Amcoff P, Kallner A, Ekman S. Vitamin C plasma concentrations and leg weakness in the forelegs of growing pigs. J Vet Med A Physiol Pathol Clin Med. 2001;48(3):165-78.

25. Massadeh AM, Al-Safi SA, Momani IF, Alomary AA, Jaradat QM AlKofahi AS. Garlic (Allium sativum L.) as a potential antidote for cadmium and lead intoxication: cadmium and lead distribution and analysis in different mice organs. Biol Trace Elem Res. 2007;120(1-3):227-34.

26. Reddy GD, Reddy AG, Rao GS, Kumar MV. Pharmacokinetic interaction of garlic and atorvastatin in dyslipidemic rats. Indian Pharmacol. 2012;44(2):246-52. 